\title{
Comparing the Roles of Representations in Face to Face and Online Collaborations
}

\author{
Daniel D. Suthers, Christopher D. Hundhausen, and Laura Girardeau \\ Laboratory for Interactive Learning Technologies \\ Department of Information and Computer Sciences \\ University of Hawai i at Manoa \\ http://lilt.ics.hawaii.edu/ \\ \{suthers,hundhaus,girardea\}@hawaii.edu
}

\begin{abstract}
This paper reports an empirical study comparing the roles of representations in supporting face to face versus distance collaborative learning. Findings indicate that while face to face collaborators primarily use knowledge representations to summarize verbal reasoning, distributed collaborators also use knowledge representations to propose ideas before they are discussed, particularly ideas supported by the categorical affordances of the knowledge representation medium. The work suggests that designers of online learning environments should support fluid crosstalk between multiple representations, because online discussions will be distributed across all available representations
\end{abstract}

\section{1: Introduction}

Online collaborative learning intrinsically requires that learning be mediated by external representations. These representations may include discourse representations such as the chat rooms and threaded discussion tools by which learners and teachers communicate in their native language [2], disciplinary representations such as visualizations and designed artifacts [4, 5], and knowledge representations such as symbolic representations of one's theories and reasoning [8]. Unlike the spoken discourse of proximal collaboration, the discourse in distance collaboration takes place in a software-supported representational medium. Given the total reliance on external representations in online collaborative learning, it is appropriate to ask how these representations should be designed to facilitate collaboration. Of particular interest is coordination between the different types of representations listed above $[1,3,13]$.

Our prior work on external representations in face-toface collaborative learning situations has shown that differences between representational notations can translate into differences in the focus of learners' discourse and collaborative activities $[6,9,10]$. As a first step towards understanding how representations might influence collaboration in distance collaboration situations, we undertook a study of how learners' use of representations changes between face-to-face and online collaboration. Specifically, the study reported in the present paper compares Proximal (face to face) with Distal (synchronous collaboration via networked software) conditions. We considered two divergent hypotheses: (1) The influence of representations in the Distal condition will be weaker because of the lack of implicit "taken as shared" meaning that results from working together in front of a physically shared display, and because of the greater difficulty of utilizing the representations as a resource for conversation through gestural deixis (identifying referents in the extralinguistic context by pointing). (2) The influence of representations in the Distal condition will be stronger because participants may rely more on them for their communication in the absence of face-to-face communication. Our results showed that the distribution of activity was quite different between the Proximal and Distal groups, and that this distribution provides evidence of both predicted influences.

\section{2: Method}

We compared the activity of participants in face-toface collaboration (Proximal) to the activity of participants in synchronous distance collaboration (Distal). Both groups were given the identical task of exploring an unsolved "challenge problem"-presented as a series of textual web pages - by recording data, hypotheses, and evidential relations in a graphical knowledge representation as they encountered them.

\section{1: Participants}

We recruited 20 students in self-selected, same-gender pairs, out of introductory biology, chemistry, physics, and computer science courses at the University of Hawai $i$. Participants were all under 25 years of age and were native English speakers. 


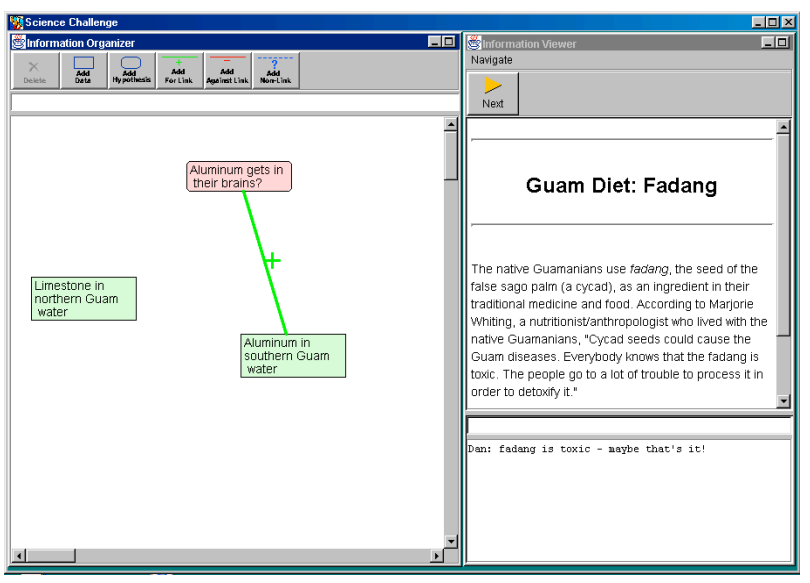

Figure 1. Collaboration Interface

\section{2: Materials}

Pairs of participants used one of two different versions of software for representing data, hypotheses, and evidential relations. The Distal version is shown in Figure 1. Participants used the upper right hand window to move forwards through a sequence of 15 pages that presented information relating to the cause of a mysterious neurological disease on the island of Guam. The left-hand window contained a graphical tool for constructing representations of the data, hypotheses, and evidential relations participants gleaned from the information pages on the right. The Graph tool is based on Belvedere [8], and enables one to build a graph of nodes expressing data items and hypotheses, and links labeled "+,", “-," or "?" representing evidential relations.

The software for the Distal condition provided a simple chat tool in an additional window in the lower right (Figure 1). Messages typed into a chat entry box were sent to both participants' shared chat displays once the message was completed and the "send" button was pressed. Both versions of the software support gestural deixis (pointing) by causing the color of objects to change when one passes the cursor over them, enhancing the ability to use the representation as a resource during conversation. The Distal version of the software replicated these color changes to the remote display.

\section{3: Procedure}

Participants first worked on a warm-up problem (on mass extinctions), which was completely unrelated to the main problem, so that they could become acquainted with the software and the information-recording process. After 15 minutes, participants were instructed to stop work on the warm-up problem, and to move on to the main problem (on a neurological disease). Participants were given as much time as they needed to explore all 15 pages on the main problem in linear order (one could not go back to previous pages). Following the learning session, participants were given 20 minutes to individually complete a multiple-choice post-test, and 30 minutes to collaboratively write an essay that discussed their hypotheses and the evidence for and against them.

\section{4: Coding of Data}

As we expected due to the short treatment times, initial post-test and essay analyses did not reveal significant differences. In this paper we focus on a categorical analysis of verbal and representational activity.

Video/audiotape of the proximal sessions were transcribed by hand. The software generated transcripts of the distal sessions automatically. Transcripts were divided into "segments," each consisting of a verbal or typed utterance (multi-propositional utterances were divided into individual segments) or a change to the representation. Then we performed a content analysis of participants' learning processes by coding all segments in the 20 transcripts into mutually exclusive "topic" categories, including the following:

- Evidential relation: indicating whether a data item supports or conflicts with a hypothesis.

- Epistemic classification: classifying information as either empirical or theoretical — that is, as either data or hypothesis.

- Hypothesis statement: stating a hypothesis concerning a possible explanation for the disease without classifying it as a hypothesis.

- Domain talk: addressing the domain of the science problem that participants are exploring.

- Off-task: segments unrelated to participants' learning task.

In addition, we coded topic segments with "modifier" categories, according to whether they were:

- Verbal or representational: spoken (in Proximal) or expressed in the chat tool (in Distal), versus represented using the software (in either condition).

- $\quad$ Recited or non-recited: quoted verbatim from the information pages, or not quoted;

- Introduced or repeated: the first occurrence of an idea within a given conversation, or a reintroduction of an idea already brought up within a given conversation.

See [9] for other categories and further details of coding.

The third author coded all of the data, while the second author coded $20 \%$ of the data. Overall agreement between the two coders ranged from $89 \%$ for the Proximal data to $95 \%$ for the Distal data, with kappa statistic values ranging from 0.86 (Proximal) to 0.94 (Distal). 
To appear in Proceedings of the International Conference on Computers in Education, December 3-6, 2002, Auckland.

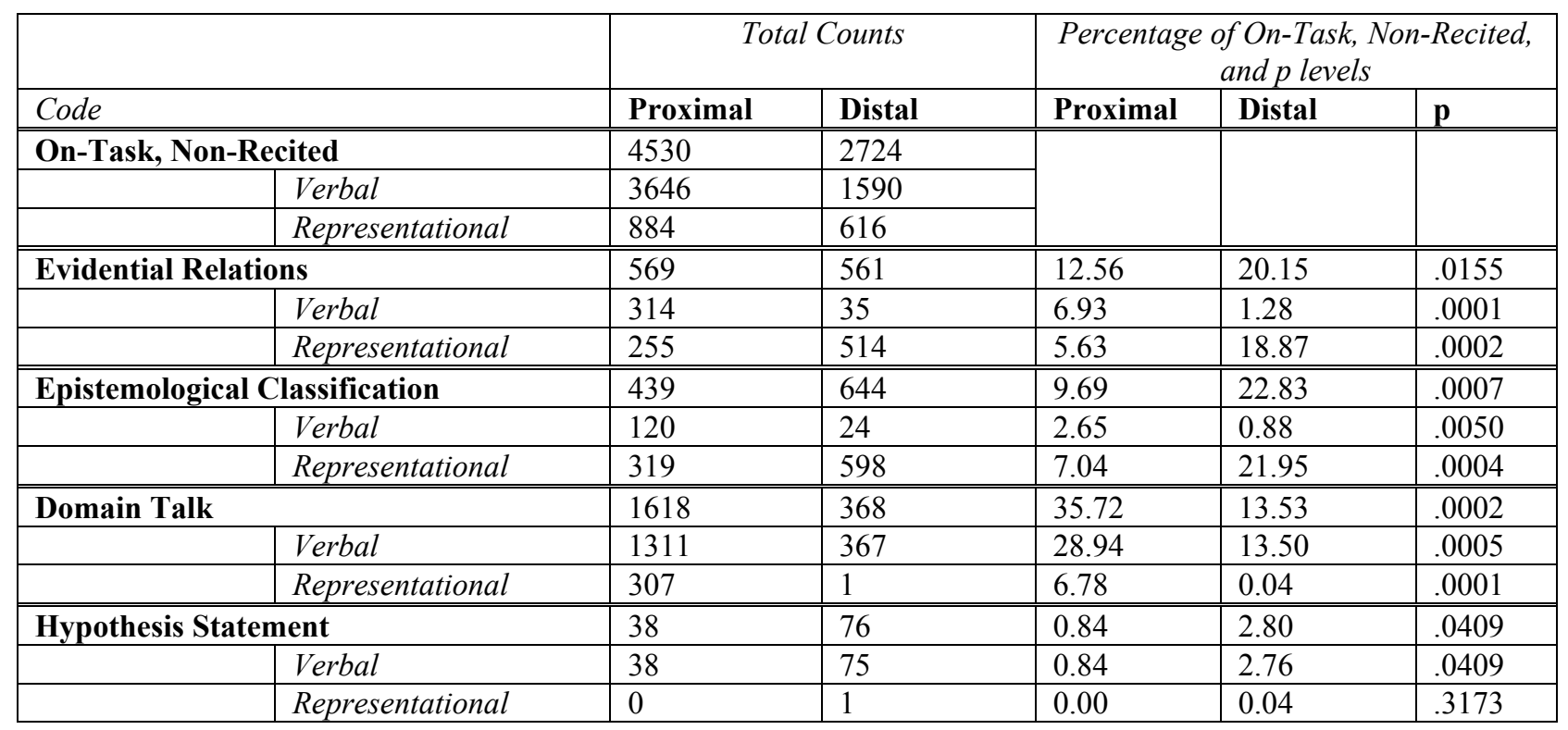

Table 1. Breakdowns of Evidential and Epistemological Classification Acts

\section{3: Results and Discussion}

\section{1: $\quad$ Statistical Tests}

Table 1 shows the categorical breakdown of On-Task, Non-Recited segments as both raw counts and percentages. Each topic category we analyzed (Evidential Relation, Epistemological Classification, Domain Talk, and Hypothesis Statement) is broken down further into Verbal and Representational. (There were 4798 segments in Proximal and 2898 in Distal: thus 268 Proximal and 174 Distal segments were either Recited or Off-task, and are not included in the analysis. Note that some categories overlap; therefore sums may not match.)

We compared each Proximal/Distal pair of percentages shown in the table using a Kruskal-Wallis ANOVA provided by Analyze-It ${ }^{\mathrm{TM}}$. (We performed statistical tests on percentages rather than raw counts to control for differences in verbosity between groups. A nonparametric Kruskall-Wallis test was used because the assumptions of the parametric ANOVA are violated when comparing ratios with varying denominators.) As indicated in Table 1, differences between all of the values shown except for the last row are statistically significant at levels ranging from $\mathrm{p}=.0001$ to .05 .

\section{2: Distribution of Categories}

The statistical results indicate that the activity as measured by our categories is quite different between the
Proximal and Distal conditions. In order to understand and explain these statistical differences, we note the following patterns in the data.

Quantity of Activity. As measured by our segment breakdown, there is a great deal more activity in Proximal than in Distal, reflecting the greater ease of speaking (Verbal acts in Proximal).

Evidential Relations. A greater percentage of acts in the Distal condition were concerned with categories provided by the Graph representations (Epistemological Classification and Evidential Relations). In the case of Evidential Relations, the counts were similar, so this reflects the difference in the denominator (the smaller number of On-Task, Non-Recited segments in Distal). Apparently the same amount of evidential thinking is taking place in context of less overall talk. However, a greater number of the Distal Evidential relation acts are classified as "Introduced" (not shown in the table), that is, are being proposed for the first time. Given that many of these acts are representational (see paragraph on interaction below), Distal participants may be using the Graph medium to propose evidential relations. Thus, the representational medium becomes part of the conversational medium, a point to which we will return.

Epistemological Classification. Both numerically and in percentage, there were many more Epistemological Classifications in Distal. This result is also consistent with the interpretation that new objects are being created in the Graph representation to propose ideas (each object creation event is also coded as a classification event). 
Domain Talk. There was more Domain talk in the Proximal condition. Domain Talk made up nearly five times more of total Proximal utterances than Distal chat. We speculate that participants are less willing to attempt to express the concepts and complex propositions of an unfamiliar domain in Chat than they are to try to express them verbally.

Hypothesis Statement. This category was observed twice as often in Distal discourse, in an apparent contradiction to the claim that content discussions are more difficult in Chat. Most Distal Hypothesis Statement tends to occur after the last page of the online materials, when participants are asked to come to a final conclusion. We speculate that Distal participants may be making up for their lack of Domain Talk in this final phase.

Interaction of Representation use with Condition. A shift from Verbal to Representational is seen when comparing Proximal and Distal, but primarily in those categories supported by the Graph representation (i.e., in Epistemological Classification and Evidential Relation but not Domain Talk or Hypothesis Statement). Participants in the Distal study represented the Epistemological Classification and Evidential Relation categories about two times more than Proximal participants. However, Proximal participants verbally discussed Epistemological Classification nearly five times more than Distal participants, and Evidential Relation categories over nine times more than Distal participants. All of these numerical differences are reflected in the percentages. This pattern indicates that distal collaborators rely more on the categorical affordances of the Graph representation to perform these kinds of acts, even though they could have just as well been expressed verbally in the Chat. Again we view these results as indicative that graphical representations are appropriated as a medium of conversation in Distal.

\section{3: Sequential Patterns of Activity}

Informal examination of typical selections from the transcripts provides additional support for our interpretation that Graph played a more conversational role in Distal. For Proximal participants, collaboration and agreement tended to be forged before graphical representations were made. The Graph representations were treated as an external product of the discourse. For Distal participants, on the other hand, the Graph representation served multiple purposes. As in the Proximal condition, Graphs functioned as a stimulus to and product of discourse. Additionally, they were used as part of the discourse medium themselves. Participants often proposed new items or relations by creating them in the Graph medium, whereupon Chat activity focused on approval or disapproval. Also, participants used the Graph in place of the Chat tool to send a message that was deleted. The Graph was also used in a manner peripheral to discourse, when a participant independently modified the Graph amidst unrelated Chat discussion. Often, Distal discourse related to representations occurred at the end of the session after all representations had been made and participants were urged to come to a final conclusion, or immediately after each representation, with short, superficial comments such as "Looks good" or "Okay."

The referencing of knowledge representations was another issue affected by discourse mode. Coordination between discourse mode and diagrammatic media was easier for Proximal participants because they could nonverbally reference items for clarity. They simply pointed to an item on the screen or clicked their cursor when determining a relationship or discussing an issue. This ability to easily reference items and determine that they were both focusing on the same items may have allowed them to discuss more items and relationships. Distal participants, on the other hand, rarely referenced items that had been previously represented, except for those that had just been added. In that case, discourse often involved simple agreements with the change in statements like "Looks good."

\section{4: Conclusions}

We began this study hoping to learn more about the differences between proximal and online collaboration before we launch into full-scale studies of online collaboration. Two hypotheses were considered: (1) that visual knowledge representations would play less of a role in guiding discourse because without co-presence they do not as easily function to convey "taken as shared" information or to support gestural deixis; (2) that visual knowledge representations would play a greater role in supporting discourse because participants would make use of them to make up for the reduced bandwidth of the verbal modes of interaction. Paradoxically, both of these seem to be supported by the study.

The first hypothesis is difficult to address without a comparative study involving Matrix and Text representations (as in [9.10]), to see whether the pattern of results changed. However, we have evidence for this hypothesis in the form of observed disconnects between the activity in the Graph and verbal Chat activity in Distal. Many uses of the Graph representations are not as tightly coupled to verbal discourse as in the Proximal case, so we would expect that their influence on verbal discourse would be weaker.

We feel we have ample evidence for the second hypothesis. A greater percentage of acts in the Distal condition were concerned with categories supported by the Graph software (Evidential Relation and Epistemological Classification), and a greater percentage of these types of acts were performed in the representational medium. Furthermore, the Graph medium appears to be used as the medium through which 
discourse takes place in addition to being the topic of discourse: ideas are often first proposed in the Graph medium and then subsequently discussed. In contrast, Proximal collaborators tended to discuss and agree on changes to the Graph representation before carrying out those changes.

Perhaps the major conclusion to be drawn from this study for the design of software for online learning is that close attention must be paid to the coordination (both in the design and in use) between multiple representations. If users are able to modify more than one type of representation, the discourse process will not be confined to the medium provided for discourse: it will be distributed across all mutable representations. Therefore the software should support fluid crosstalk between all representations by making the relationships between different representations and between acts on those representations clear. Improving linkages between representations is the subject of recent work in our laboratory $[7,11,12]$ as well as of work by others $[1,3$, $13]$.

Limitations of the study include the artificial task and laboratory setting and the short duration of participants' work with the representations. Further research should seek to corroborate our results in more authentic settings and explore the effects of variables such as representation type, task domain, asynchronous versus synchronous interaction, and different student populations. Of particular interest to this conference is the question of whether the effects reported vary between cultures in a systematic manner.

\section{5: Acknowledgements}

We are grateful to Bin Ma and Hongli Xiang for their development of the software used in this research. The National Science Foundation supported this work with grants 9873516 and 0093505 . Any opinions, findings, and conclusions or recommendations expressed in this paper are those of the authors and do not necessarily reflect the views of the National Science Foundation.

\section{6: References}

[1] Ainsworth, S.E. , Bibby, P.A. , \& Wood, D.J . (1998) Analysing the Costs and Benefits of Multi-Representational Learning Environments in Spada, H., Reimann, P. Bozhimen, \& T. de Jong (Eds) Learning with Multiple Representations, Elsevier Science, pp 120-134.

[2] Herring, S.C. (1999, January). Interactive coherence in CMC. In Proceedings of the 32nd Hawai' $i$ International Conference on the System Sciences (HICSS 32). (CDROM). Maui, Hawai'i: Institute of Electrical and Electronics Engineers, Inc. (IEEE).
[3] Hoadley, C. \& Enyedy, N. (1999). Between Information and Communication: Middle Spaces in Computer Media for Learning. In Proceedings of the Computer Support for Collaborative Learning (CSCL) 1999 Conference, C. Hoadley \& J. Roschelle (Eds.) Dec. 12-15, Stanford University, Palo Alto, California. Mahwah, NJ: Lawrence Erlbaum Associates, pp. 242-251.

[4] Hundhausen, C. D., \& Douglas, S. A. (In press). Low fidelity algorithm visualization. Journal of Visual Languages and Computing.

[5] Schank, P., \& Kozma, R. (in press). Learning Chemistry Through the Use of a Representation-Based Knowledge Building Environment. Journal of Educational Multimedia and Hypermedia.

[6] Suthers (2000), Initial Evidence for Representational Guidance of Learning Discourse. Proceedings of International Conference on Computers in Education, November 21-24, 2000, Taipei, Taiwan

[7] Suthers, D. (2001). Collaborative Representations: Supporting Face to Face and Online Knowledge-building Discourse. Proceedings of the 34th Hawai $i$ International Conference on the System Sciences (HICSS-34), January 36, 2001, Maui, Hawai i, (CD-ROM), Institute of Electrical and Electronics Engineers, Inc. (IEEE). Preprint available at http://lilt.ics.hawaii.edu/lilt/research/pubs.html

[8] Suthers, D, Connelly, J., Lesgold, A., Paolucci, M., Toth, E., Toth, J., and Weiner, A. (2001). Representational and Advisory Guidance for Students Learning Scientific Inquiry. In K. D. Forbus and P. J. Feltovich (Eds.) Smart Machines in Education: The Coming Revolution in Educational Technology. Menlo Park: AAAI Press, 2001, pp. 7-35.

[9] Suthers, D. \& Hundhausen, C. (2001). Learning by Constructing Collaborative Representations: An Empirical Comparison of Three Alternatives. In P. Dillenbourg, A. Eurelings, K. Hakkarainen (Eds.) European Perspectives on Computer-Supported Collaborative Learning, Proceedings of the First European Conference on Computer-Supported Collaborative Learning, Universiteit Maastricht, Maastrict, the Netherlands, March 22-24 2001, pp. 577-584.

[10] Suthers, D. \& Hundhausen, C. (2002). The Effects of Representation on Students' Elaborations in Collaborative Inquiry, in Proceedings of Computer Support for Collaborative Learning (CSCL) 2002 Conference, Boulder, Colorado, January 7-11, 2002, pp.472-480.

[11] Suthers, D. \& Xu, J. (2002). Kukakuka: An Online Environment for Artifact-Centered Discourse, Education Track of the Eleventh World Wide Web Conference $(W W W$ 2002), Honolulu, May 7-11, 2002, pp.472-480. Available: http://www2002.org/CDROM/alternate/2521

[12] Takeda, T. \& Suthers, D. (2002). Online Workspaces for Annotation and Discussion of Documents. To appear in Proceedings of the International Conference on Computers in Education, December 3-6, 2002, Auckland.

[13] Turoff, M., Hiltz, S. R., Bieber, M., Fjermestad, J., \& Rana, A. (1999). Collaborative discourse structures in computer mediated group communications. Journal of Computer Mediated Communication, 4(4). Available: http://jcmc.huji.ac.il/ 